\title{
The qualification "primary" can only be allocated on the basis of metrological criteria
}

\author{
Paul De Bièvre
}

Published online: 3 March 2015

(C) Springer-Verlag Berlin Heidelberg 2015

The use of the qualifier "primary" is ubiquitous in the chemical literature: "primary reference measurement procedure" or "primary reference procedure" (entry 2.8 in [1]), or "primary measurement standard" ("primary standard" for short-entry 5.4 in [1]) are considered to have a high scientific status. Thus, they are used frequently. The qualifier "primary" is thereby allocated easily, rather loosely, and not necessarily on the basis of metrological criteria.

A measurement standard (of which a certified reference material is a typical example in chemistry) usually is a material carrying a property value or having a property value embodied in the material. It is that property value which will be used in, e.g., a calibration by means of this material, or which will be used for verifying the presence or absence of systematic effects in a measuring system (entry 3.2 in [1]). If there is a strong wish for such a reference material to be viewed as having a "higher status" for commercial and/or political reasons, it is often called "primary," just to increase that status. In addition, a very small or "smallest possible" measurement uncertainty for the embodied property value is implied or declared, sometimes without being consistent with the VIM definition of measurement uncertainty (entry 2.26 in [1]).

The knowledge of the property value of a chemical measurement standard usually is obtained by means of a reference measurement procedure (entry 2.7 in [1]) (a

Disclaimer: The author is a member of the Joint Committee on Guides for Metrology (JCGM), Working Group 2 (VIM). The opinions expressed in this Column do not necessarily represent the view of the Working Group or of ACQUAL.

P. De Bièvre $(\bowtie)$

Kasterlee, Belgium

e-mail: paul.de.bievre@skynet.be measurement procedure is a description of how to carry out a measurement-see entry 2.6 in [1]). Such a procedure usually consists of a series of steps or operations each of which - through its uncertainty-contributes to the uncertainty budget (entry 2.33 in [1]) of the measured property value. A case in point is the measurement of the mass fraction of Ag (the measurand) by constant current coulometry in an impure raw silver-containing material. First, a weighed portion of the raw silver material is dissolved in a weighed portion of $\mathrm{HNO}_{3}$ solution. Measuring a constant electric current passing through the solution, depositing the $\mathrm{Ag}$ on an electrode and measuring the time needed until depletion of $\mathrm{Ag}+$ ions, leads to the knowledge of the measurand (entry 2.3 in [1]) by a calculation of the number of $\mathrm{Ag}$ atoms or the mass of the Ag per $\mathrm{g}$ of the raw silver material. Note that the term "analyte" is frequently usederroneously - to designate either the pure Ag or the raw silver material which is a recipe for confusion because an analyte is not a quantity and can therefore not be a measurand.

It is also possible to arrive at the property value to be certified, by using a "preparation procedure" through which the final property value embodied in the standard is obtained. A simple example of that is the preparation of a glucose measurement standard obtained by mixing a known mass of glucose and a known mass of water, both of known (preferably high) purity. This enables to calculate a property value expressed as a measured mass of glucose per measured mass of water or per measured mass of solution, expressed in the (derived) unit $\mathrm{g} / \mathrm{g}$ for mass fraction. The end product: a g/g value or number ratio for glucose in water for which an associated uncertainty must be evaluated. It is essential to indicate the measurand (e.g., mass fraction) as well as the unit used, i.e., g/g. Once the measurement standard has been established, one can say 
that the embodied property value in the standard has been synthesized by a procedure which is totally different from the role the standard will play in any subsequent measurement procedure in which the standard is used.

Can the value embodied in a measurement standard be obtained by a routine measurement procedure using a routine measuring system for the same measurand? Or in an interlaboratory comparison?

No.

Obtaining a value by a routine measurement procedure requires a calibration (as in any measurement) by a measurement standard. When used to attain a certified value for such a measurement standard, a circular way of thinking and working develops in which a measurement standard's value would be used to arrive at a measurement standard's value for a measurement of the same measurand (see [2]). Circular procedures in science are, of course, unacceptable as a matter of principle.

Where does that leave us for the qualifier "primary"?

In the case of glucose, the certified value for the measurement standard for measuring a glucose concentration in water was attained "without relation to a measurement standard" for mass concentration measurements of glucose in water. It was obtained via a totally different and independent route. That entitles this certified value to be called "primary."

Thus, the attribution of the qualification "primary" depends on the procedure used to obtain the property value to be certified for the measurement standard. That procedure must not make use of a standard of the same kind. A logical-one could say a metro-logical-criterion determines whether the qualification "primary" can be attributed to a given measurement procedure. Thus, a property value obtained by a "preparation procedure" can be seen as equivalent to a property value obtained by a "measurement procedure." That puts the burden of justifying the attribution of the qualifier "primary" squarely on the reference material producer. If proof of that cannot be given in the certificate of the standard, that standard cannot be claimed to be a "primary measurement standard."

Because of this scientific-metrological way that the standard's certified property value is arrived at, it is seen as the "realization" of the (theoretical) definition of the measurement unit for the property involved (in the glucose example the unit $\mathrm{g} / \mathrm{g}$ ). In a metrological traceability chain (entry 2.41 in [1]), it is the first step after the definition of a unit (see chapter 9 in [3]). A measurement of the property value concerned, using another measurement standard for the same measurand, does not yield a primary measurement standard but a secondary measurement standard (entry 5.5 in [1]).

Do we have objective and intercontinentally agreed criteria as well as the necessary concepts at our disposal for the attribution of the qualification "primary"?

Yes.

They can be found in the quoted definitions from the International Vocabulary of Metrology-VIM [1]. Since the existence of this Vocabulary (2008), valid translations can be made of that Vocabulary in any other language because the translators can find in the VIM the meaning of the term to be translated, thus enabling the application of the same objective criteria to so-claimed "primary measurement standards" worldwide. An arbitrary allocation of the qualification "primary" for either prestige, political, or commercial reasons only, is not permitted since such a qualification is not a criterion for being allocated the qualifier "primary."

The reason why we take up this matter again is that little progress has been made in a better metrological underpinning of the qualifier "primary" since attention was drawn on this point 3 years ago [3].

As usual, any comment, question, or amendment is welcome, preferably as a contribution to the Discussion Forum of this Journal.

\section{References}

1. BIPM, IEC, IFCC, IUPAC, IUPAP, ISO, OIML, The international vocabulary of metrology-basic and general concepts and associated terms (VIM), edn 3, Joint Committee on Guides for Metrology, JCGM 200:2008/2012 at http://www.bipm.org/en/ publications/guides/vim.html and http://jcgm.bipm.org/vim)

2. De Bièvre P (2011) The qualifier "primary" should not be used too lightly in the context of measurement. Accred Qual Assur $16: 61-62$

3. P De Bièvre, R Dybkaer, A Fajgelj, B Hibbert (2011) Metrological traceability of measurement results in chemistry-concepts and implementation. http://iupac.org/publications/pac/83/10/1873 